\title{
MicroRNA: A Target Candidate to Turn the Tide
}

\section{Dhilleswara Rao V*}

Department of Biotechnology, Bharathidasan University, TN, India

\begin{abstract}
Understanding the molecular basis of life is at the centre today, the integration of Computer Science, Mathematics and statistics improved the belief in structural and functional genomics. Recently there is an increased interest in function of non-coding RNA transcripts. Non-coding RNAs are small molecules that are not translated into proteins, but these molecules have a pivotal role in almost all molecular and cellular processes. MicroRNA is one of those noncoding RNA molecules that regulate gene expression at posttranslational level. These 18-25 nt length molecules are primary transcribes consist of a secondary hairpin structure. There are 700 miRNAs in the human genome, at least $1-4 \%$ of expressed genes; this makes miRNAs as the biggest group of regulators. This review summarizes biogenesis, role of expression profiles in and at various cellular and molecular mechanisms and their applications. More or less, research on non-coding RNA candidates has been done and proved their importance, however, even more to be proved.
\end{abstract}

Keywords: MicroRNA; Cancer; Non-coding RNA; CSCs; Biomarker

\section{Introduction}

It has been assumed that most of the genetic material is expressed as protein in living systems; the majority of the mammalian genome is yielding complex overlapping transcripts that are known as non-coding RNAs. These transcripts represent transcriptional noise, show high degree of specification in expression, localization, and associated with diseases. Since the origin is not yet clearly evolved, some researchers found the role of MALAT1 and lack of poly(A) tail at $3^{\prime}$. MALAT1 is a highly conserved locus that localizes to nuclear speckles. This transcript is misregulate in many human cancers. Studies demonstrated that a conserved tRNA like structure present nearby $3^{\prime}$ is recognise and cleavage the MALAT1 locus at $3^{\prime}$ [1]. Despite being transcribed by RNA pol II, the $3^{\prime}$ is recognised and cleavage by tRNA likes structure by RNase P. Thus, this results in lack of poly(A) tail at $3^{\prime}$ end and shows high level expression than protein coding genes. Wilusz et al. demonstrated these long non-coding RNAs that lack of 3 ' stabilized by a triple helix structure. The study also proved that the triple helix is effectively repressed by miRNAs in vivo and the role of poly(A)in miRNA mediated splicing [2]. The noncoding RNAs shows vast range of structural diversity since few of them for example miRNAs derive from distinctive hairpin (fold back precursors) structure where as other noncoding RNAs are from long hairpin or RNA duplexes SiRNA or PiRNAs [3].

\section{Evolution and biogenesis of miRNA}

MicroRNAs are small, widely distributed and evolutionary conserved, small sequences that are transcribed and instead of translate into proteins, they regulates the expression of other genes. Human genome encoding for various types of RNAs such as tRNA, rRNAs, etc., interestingly, $95 \%$ of human genome is non-coding DNA, show vast diversity among species and associated with clinical and circumstantial malfunction [4]. MicroRNAs target both mRNA degradation and suppression of translation based on complementarities between miRNA and mRNA. There was some interesting facts reported regarding evaluation of miRNA, some RNA retroviruses penetrate in eukaryotic genome at noncoding regions and acting as transposons and provide defence themselves from viral invasion and expressed as secondary level gene modulations [4].

MiRNAs origin and functional evolution is not yet clearly understood but some studies have conducted with the aim to unravel the evolution of MiRNAs. Meunier et al. conducted a study by using RNA sequencing generated an extensive and comparable data and reported complete data. This was the first study that revealed the origin, birth and evolution of MiRNAs. The threefold birth rates of miRNA was facilitated by de novo pathway and reported that miRNAs that are originated on the X-chromosome evolved high expression levels at various cellular processes [5].

Nevertheless, it was a challenge to the researchers to find out the biogenesis of these non-coding sequences, however, series of attempts have been made to reveal the biogenesis. Kim et al. reported in animals, nuclear Drosha RNase III enzyme cleaved pre-miRNAs to release pre-miRNA haipins that are subsequently cleaved by cytoplasmic Dicer RNase III to produce miRNA duplexes [6]. In another study by Flynt et al. [7] reported the biogenesis of miRNA via Splicing and exosome mediated trimming, this research group conducted genetic and Biochemical experiments in Drosophila and defined the pathways that are involved in splicing and exosome-mediated "trimming" followed by conventional molecular mechanisms to yield miRNA. Furthermore, exosome plays key role in biogenesis of miRNA from hybrid mirtron substrates [7]. Recognition of small RNAs in animal systems was revolutionized event and provided possibilities for studies on reverse genetics. These small molecules require Dicer RNase III enzymes for biogenesis and Argonaute proteins for target silencing. In a study, Yang et al. used structural and functional analysis to define the functional parameters of Ago2-mediated miRNA biogenesis. The study showed that certain hairpin lengths competence to mature via both Dicer-mediated and Ago2-mediated Pathways, however, conventional shRNAs can traverse Dicer Independent pathway [8]. There are few evidences reported similar results in various organisms. Halic and Moazed showed Dicer- independent primal RNAs trigger RNAi and heterochromatin formation yeast [9], Lee et al. showed diverse pathways generate miRNA-like small RNAs and Dicer-independent siRNAs in filamentous fungi [10]. Cifuentes et al. reported Ago2 catalytic activity in maternal-zygotic mutants and found the Ago2

*Corresponding author: Dhilleswara Rao V, Department of Biotechnology, Bharathidasan University, TN, India, Tel: +91 8106544565; E-mail: drvana.biotech@gmail.com

Received November 02, 2015; Accepted November 11, 2015; Published November 18, 2015

Citation: Dhilleswara Rao V (2015) MicroRNA: A Target Candidate to Turn the Tide. Mol Biol 4: 145. doi:10.4172/2168-9547.1000145

Copyright: (c) 2015 Dhilleswara Rao V. This is an open-access article distributed under the terms of the Creative Commons Attribution License, which permits unrestricted use, distribution, and reproduction in any medium, provided the original author and source are credited. 
activity in vivo. This study proposed, Ago2 -mediated cleavage of PremiRNAs, followed by uridylation and trimming, generates functional miRNAs independently of Dicer [11].

Computer application in Biology seems to make it possible to isolation and identification of many sequences those function to be determined, but in mammalian cells, the pre miRNS are not in enough amounts to trigger the RISC assembly. Shi-Lung Lin et al. developed a computer based intronic-miRNA expression system to overcome the problem. This research group, for the first time shown that the intron derived miRNAs are able to induce strong RNAi effects in human, mouse, zebrafish, chicken embryos, and adult mice [12]. Another Computational approach has reported to identify miRNA in plants. Oliveira et al. proposed CRAVELA, a tool developed for miRNA discovery in animals, which is one of the single-genome microRNA finding tools and NGS data analysing algorithm, a scoring function to evaluate the expression profiles of miRNAs [13]. The group carried out the experiments using E. grandis genome assembly and provided in silico and bench procedures to analyse high-throughput data but failed to discuss the biological significance, however, the integration of Computational Biology Mathematics and informatics with genomic knowledge would be more helpful to reveal the gene functions and interactions.

\section{MiRNA in cellular and molecular processes}

Evidences supported by recent data and research miRNA are the one of the central molecules in cellular and molecular processes such as development, cell proliferation, cellular differentiation, cell cycle control and apoptosis. Cell proliferation and differentiation is a basic and complex mechanism in cellular processes [14]. The most important and best-studied differentiation is erythropoiesis mechanism; recent studies reported that numerous specific miRNAs plays a vital role in cell proliferation processes. There are thousands of miRNAs have been found in almost all the organisms and they mediate the regulation of different cellular mechanisms apoptosis, carcinogenesis apart from cell proliferation [15]. MiRNAs regulate the gene expression by two processes; one is direct degeneration and other one is translation inhibition. Past studies reported that the noncoding molecules play a key role in gene regulation of embryonic stem cells (ESC) as well. These molecules repress pluripotent mRNA genes such as Sox 2 and Nanog, etc. The available studies suggest miRNA have a critical role in pluripotent cell regulation of mammalians in early development. Berardi et al. reported in their study that these small molecules regulates the gene expression of ESCs and control the mesodermal, endodermal, exodermal differentiation and cell reprogramming as well [3].

Apoptosis or programmed cell death (PCD) regulated by external and internal factors. The equity between apoptosis and cell proliferation altered in cancer cells. Shimada [16] reported microRNA mediated regulation of apoptosis in bone cancer. Two cluster miRNAs 17-92 and $14 \mathrm{q} 32$ located at 13 and 14 chromosomes respectively regulate cell cycle, proliferation, apoptosis, and metastasis. Over expression of 17 92 clusters enhance the cell proliferation. The expression levels of this cluster are high in chronic myeloid leukemia (CML) especially, at early chronic phase, in other hand the high-level expression profiles of 17-92 cluster inhibit apoptosis and potential oncogene [17-19].

There are many external and internal factors including chemical and physical carcinogens that are highly effect the human genome. The molecular basis of life depends on critical processes like DNA replication, transcription and translation. Protecting the genome from these factors is a critical molecular mechanism for which cell induces high degree protection system such as cell cycle checkpoints,
DNA repair system collectively called DNA damage response and repair (DDR). There is experimental evidence that miRNAs likely to be involved in DDR, such as miR-24, 96, 138, and 182 have been implicated in DDR [20]. However, studies to be done to know more specifically about the roles of miRNAs in DDR system to design and development of novel therapeutics.

\section{miRNAs in cancer studies}

In past most of the studies have focused on role of coding genes in cancer, due to the recent approaches in molecular biology revolutionized miRNAs research, belief in role of non-coding RNAs has increased. In biochemical or molecular pathways regulators should be tightly regulated, microRNA roles in regulatory pathways already have been reported, few studies suggested that the percentage of identified miRNA coding genes are located at tumor related fragile chromosome regions. Remarkable achievements have been made in understanding regulation of miRNA pathways as well as functions of miRNAs in tumorgenesis and metastasis [21]. The polymorphism in miRNAs is significant in phenotypic variation and disease susceptibility [14]. As research grows, the role of CSCs and miRNAs in cancer development and regulation reported. Deregulation in the expression of both miRNAs and mRNAs in the CSC and developing therapies for cancer aggressiveness [22]. The mutations in miRNAs and polymorphism in target mRNAs contribute to cancer progression. The evolving concept showed that cancer is driven by cancer stem cells (CSCs). CSCs have self- renewal, which help them to differentiate into cancer cell that are similar to parental cell in phenotypic and genotypic aspects. Sethi and Sarkar reviewed possible roles and associations of miRNAs with cancer. The review summarized various key aspects such as miRNA relevance to CSCs, associated with human malignancy, different types of miRNAs involved in cancers, alternations, expression and clinical resistance of miRNAs to CSCs. This increase the whole picture and understanding of importance of microRNAs in cancers [23].

George et al. used computational tools to predict the functional role of miRNA-targeted mRNAs in colon cancer. In the study, they applied PupaSuite, miRBase and UTRscan to validate miRNA targets in twenty colon cancer genes and predicted $50 \%$ of miRNA targets. This is of significant and could be apply same methodology to predict various types of cancer genes [14]. Gastric cancer is one of the asymptomatic diseases, though; there are few treatment methods available but limited due to their side effects. Guo-Hua Tang et al. [24] reported the association of miRNAs and gastric cancer, mir-19a functions as an oncogenic, which represses the expression of tumor suppressor gene SOCS1. However, these miRNAs are in low quantity and stable in gastric cancers, therefore, could be considered as promising biomarker to diagnosis of gastric cancer. The expression profiles of these molecules are different in specific stages and types of gastric cancer, which is a potential target for preventions and treatment of gastric cancer.

However, colorectal cancer (CRC) is preventable when the preneoplastic lesions are deleted at early stages; morbidity and mortality from CRC represent a major health problem. Since, lack of sensitivity in existed screening methods; early detection would be more helpful if most accurate, clinically proven and cost-effective biomarkers are available. There are some protein-based biomarkers available such as carcinoembryonic antigen and carbohydrate antigen 19-9, are the best characterized among the known serum protein markers, however, there are limitations likely to make them unavailable for screening of CRC. In addition to protein biomarkers, there are some nucleic acid based biomarkers also reported, however, Yung-Bin et al. proved that these markers to be passed the validation and confirm the ability in 
early diagnosis of CRC [25]. Studies have indicated that dysregulation of miRNAs in CRC associated with pathological pathways of tumor development and stability of these molecules associated with their diseases-specific profiles suggested that miRNAs are promising biomarkers for gastrointestinal and CRC screening at early stage $[25,26]$. Though it has proven the ability of miRNAs in screening tests; there are few issues to be fixed before applying in clinical practices, for instance, low sensitivity of miRNAs, which is a difficult process to amplify the short target RNA transcripts and sometimes leads falsepositive results from closely related miRNA precursors [25-27].

Diversification expression profiles of miRNAs tightly linked to cancers, according to past studies $15-30 \%$ of the mutations in cancer activated by miRNAs. These expression profiles are used for classification, diagnosis and progression of human cancers. Earlier, we reviewed and concluded with roles of various miRNA expression profiles and their significance as biomarker in respective cancer types [28]. The abilities of these non-coding sequences as biomarker are reported many cancers such as, biomarker in progression of cervical cancers [29], miR-100 in bladder carcinomas [30], transcriptionally induced and anti-cancer mechanism of miR-98 in prostate cancer [31], serum microRNA as potential biomarker for breast cancer [32]. Salivary miRNA roles in detection of oral cancer [33], positive and negative regulation of miRNA play a key role in detection and progression of liver cancer [34].

Hence, many efforts made to diagnosis, and treatment, prevention of lung cancer, the survival rate is very poor that shows the necessity of an early detection biomarker. Hubaux et al. presented many targets for lung cancer association with miRNAs through miRNAregulated pathways. Let-7 and miR17-91 are involved in cell cycle regulation and process of lung cancer [35]. Evidences unveil different regulation and expression levels of miRs that are closely associated with cancers; however, their clinical significance was reported as well, for instance emerging roles of miRNA has reported, over-expression or down-regulation of miRNAs play critical roles in PC progression, moreover, 37 miRNAs were decreased and 54 miRNAs were increased in the plasma of PC patients. Upregulation of miR-103 and 107, downregulation miR-155 are helpful to identify pancreatic tumors and miR21, 29a, 92, 93 and 126 were upregulated whereas miR-155, 99b, 27 are down regulated in ovarian cancer [36-39]. The cluster of miRNAs and their expression profiles likely to enhance cell proliferation and cell death in common cancers like Osteosarcoma, lung cancer and chronic myeloid leukemia was reported [16-19].

\section{Clinical significance of miRNAs}

Despite, the roles are unclear, the origin not yet clearly understood, miRNAs are now more significant biological target for clinicians as well. Their role was briefing out above in cellular and molecular aspects but they are not limited as a target biomarker. Apart from oncologists or molecular biologists, these molecules are targets for cardiologists, clinicians as well. In our previous review, mentioned few cases as biomarker for cardiologists, hereby, this study summarizes the cuttingedge applications of miRNA.

It has become a challenge to cardiologists for cell therapy applications since cardiac progenitor cells have limited regeneration capacity. Loperfido et al. reported an association between miRNA expression and cardiac progenitors. Deregulated miR-669 could aberrant differentiation of CPCs both in vivo and in vitro. Most of the pathologies are associated with expression profiles of miRNAs; furthermore, in presence of miRNAs, the regeneration or reprogramming of CSCs were attained 3D structures of cardiac muscle in correct orientation.
In a recent study by Serradifalco et al. proved that nanoparticle bearing miRNAs reprogrammed CPCs in a biodegradable 3D scaffold [40,41]. Now, these molecules have great potential to understanding the pathophysciology and offer a novel class of biomarkers for acute myocardial infarction diagnosis. Fortunately, this has been reported in many studies that a miRNA based signature that consist of 20 miRNAs, can predict the acute myocardial infarction sensitivity, accuracy and specificity with $90 \%, 93 \%$ and $96 \%$ respectively. This shows the future hope of miRNAs as biomarkers $[42,43]$. Based on recent data obtained miRNAs act as intercellular and intracellular mediator in cardiac diseases could be considered as prognostic biomarker, moreover, the potential usefulness of cardiomyocyte-enriched miRNA as diagnostic marker in acute coronary syndrome and the contribution of miRNAs (miR-21) as target in heart failure resulting in novel therapeutic strategies [44-46].

Recently, circulating miRNAs attracted the interest due to the abundance of RNases in circulating blood; therefore, miRNAs need a protection mechanism from degradation. Microparticles are one of these protection mechanisms that can transport large number of miRNAs, which are associated with cardiovascular diseases. The expression profiles in CAD, ACS already investigated in vivo and in vitro. The miRNA profiles in circulating cell and plasma are differ in between ST-elevation myocardial infarction (STEMI), Non STelevation myocardial infarction (NSTEMI) $[47,48]$.

More or less, the molecules have significant importance in all clinical applications. There are few obstacles in targeted drug delivery there were clinical trials with mesenchymal stem cell (MSC) that are highly promising targeted drug delivery of RNAi. There is no unexpected reported yet, however, to deliver the RNAi safely carful experimental procedures are required [49]. The use of MSCs in RNAi therapeutics would be a breakthrough in cancer treatment, but need to be understood the biology of MSC and their affinity towards the site of cancer and tissue damage as well $[49,50]$. In addition, the regulatory networks of miRNAs provide feedback mechanisms for steroid receptor signalling. Activation of steroid receptors miRNA expression by direct interactions with gene promoters of $\mathrm{miR}$, in contrast, miRNAs may regulate the critical pathways or extract regulatory effects on steroid receptors [51]. This could be improving future findings of therapeutic or prognostic biomarker for horomone related disorders.

Furthermore, these molecules have prominent role as non-invasive biomarker for various diseases. It has been reported that the potential utility of urinary miRNA as sensitive and more consistent biomarker for drug induced liver injury [52,53]. Lee et al. reported the roles of miRNA in cellular therapy of Ischemia [54]. The research group developed a novel biosensor for imaging endothelial precursor cells treated therapy of Ischemia, which could be used for in vivo and in vitro imaging of miRNA that can monitor cellular developments and diseases treatments. Al-Shorafa and Sharif [55] reported miR role in recurrent pregnancy loss (RPL) in a case of 21 years old woman. In this case they have studies relative expressions of miR-21, miR-126, miR-155, miR-182, miR-222 and miR-517. The woman lost her fetus at the end of eight week of her pregnancy due to dysregulation miRNAs involved in RPL [55].

\section{Conclusions and future prospective}

However, we summarized recent data, in our review, mainly focused on cancer diagnosis and treatment applications of miRNA. Immense research is going on to reveal the expression panels of miRNA, which enhances the identification and understanding aggressive diseases and therefore, provide better treatment and therapeutic development 
strategies. Many studies proved that miRs are novel, potential, noninvasive targets for prevention and treatment of cancers, in addition, their ability in early diagnosis also reported. Though there are enough evidences, the miRNA related approaches in clinical applications should be relatively high specific, less in adverse events, non-toxic eventually, inexpensive to synthesize $[24,29,56]$. There are many hurdles to overcome the identification and expression profiles of miRNAs by using traditional methods, which clearly indicates the importance of new tools that identify the significant fold changing and association with disease at group levels such as miRNA set approach, which uncovers chronic heart failure in miRNA microarray analysis [57]. The belief in boom of computational and systems biology, bioinformatics may enable the identification which is far from being understood completely.

As discussed earlier, miRNAs need to be passed clinical validation, for which well-designed protocols and adequate sample sizes are required. MiRNA marker panels, combinations of several miRS, can enhance diagnostic performance, to fulfil this systematic tests are required. "Considering the fact that miRNAs are involved in nearly every cellular process, the identification and characterization of small molecule modulators of RNAi/miRNA pathway will provide novel insights into the fundamentally pathological mechanisms of human diseases particularly cancer. Furthermore, these RNAi modulators, particularly RNAi enhancers, could potentially facilitate the development of RNA interference as a tool for biomedical research and therapeutic interventions" [21,25]. Intensive study and continuous research on non-coding molecules, particularly on miRNAS will be answered the developments and they turn the tide.

\section{References}

1. Wilusz JE, Freier SM, Spector DL (2008) 3' end processing of a long nuclearretained noncoding RNA yields a tRNA-like cytoplasmic RNA. Cell 135: 919932.

2. Wilusz JE, JnBaptiste CK, Lu LY, Kuhn CD, Joshua-Tor L, et al. (2012) A triple helix stabilizes the 3 ' ends of long noncoding RNAs that lack poly $(A)$ tails. Genes Dev 26: 2392-2407.

3. Berardi E, Pues M, Thorrez L, Sampaolesi M (2012) miRNAs in ESC differentiation. Am J Physiol Heart Circ Physiol 303: H931-939.

4. Ying SY, Chang DC, Lin SL (2013) The MicroRNA. Methods Mol Biol 936: 1-19.

5. Meunier J, Lemoine F, Soumillon M, Liechti A, Weier M, et al. (2013) Birth and expression evolution of mammalian microRNA genes. Genome Res 23: 34-45.

6. Kim VN, Han J, Siomi MC (2009) Biogenesis of small RNAs in animals. Nat Rev Mol Cell Biol 10: 126-139.

7. Flynt AS, Greimann JC, Chung WJ, Lima CD, Lai EC (2010) MicroRNA biogenesis via splicing and exosome-mediated trimming in Drosophila. Mol Cell 38: 900-907.

8. Yang JS, Maurin T, Lai EC (2012) Functional parameters of Dicer-independent microRNA biogenesis. RNA 18: 945-957.

9. Halic M, Moazed D (2010) Dicer-independent primal RNAs trigger RNAi and heterochromatin formation. Cell 140: 504-516.

10. Lee HC, Li L, Gu W, Xue Z, Crosthwaite SK, et al. (2010) Diverse pathways generate microRNA-like RNAs and Dicer-independent small interfering RNAs in fungi. Mol Cell 38: 803-814.

11. Cifuentes D, Xue H, Taylor DW, Patnode H, Mishima $Y$, et al. (2010) A nove miRNA processing pathway independent of Dicer requires Argonaute2 catalytic activity. Science 328: 1694-1698.

12. Lin SL, Chang DC, Ying SY (2013) Isolation and identification of gene-specific microRNAs. Methods Mol Biol 936: 271-278.

13. Oliveira JS, Mendes ND, Carocha V, Graça C, Paiva JA, et al. (2013) A Computational Approach for MicroRNA Identification in Plants: Combining Genome-Based Predictions with RNA-Seq Data. J Data Mining Genomics Proteomics 4:130.
14. George PDC, Dike IP, Rao S (2008) Application of Computational Tools for Identification of miRNA and Their Target SNPs. J Proteomics Bioinform 1: 359 367

15. Listowski MA, Heger E, BogusÅ,awska DM, Machnicka B, Kuliczkowski K, et al (2013) microRNAs: fine tuning of erythropoiesis. Cell Mol Biol Lett 18: 34-46.

16. Shimada M (2013) MicroRNA-Mediated Regulation of Apoptosis in Osteosarcoma. J Carcinogene Mutagene S6:001. doi: 10.4172/2157-2518. S6-001.

17. Hayashita Y, Osada H, Tatematsu Y, Yamada H, Yanagisawa K, et al. (2005) A polycistronic microRNA cluster, miR-17-92, is overexpressed in human lung cancers and enhances cell proliferation. Cancer Res 65: 9628-9632.

18. Venturini L, Battmer K, Castoldi M, Schultheis B, Hochhaus A, et al. (2007) Expression of the miR-17-92 polycistron in chronic myeloid leukemia (CML) CD34+ cells. Blood 109: 4399-4405.

19. He L, Thomson JM, Hemann MT, Hernando-Monge E, Mu D, et al. (2005) A microRNA polycistron as a potential human oncogene. Nature 435: 828-833.

20. Wang $\mathrm{Y}$, Taniguchi $\mathrm{T}$ (2013) MicroRNAs and DNA damage response: implications for cancer therapy. Cell Cycle 12: 32-42.

21. Jiang R, Li Y (2013) Regulation of Mirna Pathway and Roles of Micrornas in Tumorigenesis and Metastasis. Human Genet Embryol S2:007

22. Ahmed A, Ali S, Philip PA, Sarkar FH (2012) The Role of Cancer Stem Cells and MicroRNAs in the Development and Progression of Pancreatic Cancer. J Stem Cell Res Ther S7.

23. Sethi S, Sarkar FH (2011) Evolving Concept of Cancer Stem Cells: Role of Micro-RNAs and their Implications in Tumor Aggressiveness. J Carcinogene Mutagene S1:005.

24. Tang GH, Tang M, Xie YJ (2013) The Role of miRNAs in Gastric Cancer. J Gastroint Dig Syst 3:129.

25. Yung-Bin K, Err-Cheng C, Jinn-Shiun C, Fa-kuen S (2013) Fecal miRNAS as Biomarkers for the Detection of Colorectal Cancer. J Gastroint Dig Syst S12:016.

26. Ahmed FE, Jeffries CD, Vos PW, Flake G, Nuovo GJ (2009) Diagnostic microRNA markers for screening sporadic human colon cancer and active ulcerative colitis in stool and tissue. Cancer Genomics Proteomics 6: 281-295.

27. Ahmed FE (2007) Role of miRNA in carcinogenesis and biomarker selection: a methodological view. Expert Rev Mol Diagn 7: 569-603.

28. Rao VD (2013) MicroRNA: A New Promising Biomarker. J Cancer Sci Ther 5:066-068.

29. McBee WC Jr, Gardiner AS, Edwards RP, Lesnock JL, Bhargava R, et al. (2011) MicroRNA Analysis in Human Papillomavirus (HPV)-Associated Cervical Neoplasia and Cancer. J Carcinogene Mutagene 1:114

30. Dip N, Reis ST, Timoszczuk LS, Abe DK, Dall'Oglio M, et al. (2012) Underexpression of miR-100 may be a new Carcinogenic pathway for low-grade pTa Bladder Urothelial Carcinomas. J Mol Biomark Diagn 3:121.

31. Ting HJ, Messing J, Yasmin-Karim S, Lee YF (2013) Identification of microRNA-98 as a therapeutic target inhibiting prostate cancer growth and a biomarker induced by vitamin D. J Biol Chem 288: 1-9.

32. Sun Y, Wang M, Lin G, Sun S, Li X, et al. (2012) Serum microRNA-155 as a potential biomarker to track disease in breast cancer. PLoS One 7: e47003.

33. Yoshizawa JM, Wong DT (2013) Salivary microRNAs and oral cancer detection Methods Mol Biol 936: 313-324.

34. LeBrun DG, Yu X, Li M (2011) The Future of MicroRNAs in Liver Cancer. J Liver 1:e101.

35. Hubaux R, Becker-Santos DD1, Enfield KS1, Lam S1, Lam WL1, et al. (2012) MicroRNAs As Biomarkers For Clinical Features Of Lung Cancer. Metabolomics (Los Angel) 2: 1000108.

36. Xia J, Sarkar FH2, Wang Z3 (2012) Emerging Role of MicroRNA in Pancreatic Cancer. Pancreat Disord Ther 2: e114.

37. Ali S, Almhanna K, Chen W, Philip PA, Sarkar FH (2010) Differentially expressed miRNAs in the plasma may provide a molecular signature for aggressive pancreatic cancer. Am J Transl Res 3: 28-47.

38. Roldo C, Missiaglia E, Hagan JP, Falconi M, Capelli P, et al. (2006) MicroRNA expression abnormalities in pancreatic endocrine and acinar tumors are 
associated with distinctive pathologic features and clinical behavior. J Clin Oncol 24: 4677-4684.

39. Cheng F (2012) Blood MicroRNAs: Novel 'Omics' Biomarkers for Ovarian Cancer Early Detection. J Proteomics Bioinform 5: xx-xxi.

40. Loperfido M, Crippa S, Sampaolesi M (2012) miRNA Lentiviral Vector Integration and Gene Targeting Efficacy in Cardiac Progenitors. J Stem Cell Res Ther S9:003.

41. Serradifalco C, Zummo G, Felice VD (2012) MicroRNA and Cardiac Stem Cell Therapy. J Clin Exp Cardiolog S11:001.

42. Shahzad A, Yin X, Sami M, Hou D (2012) MicroRNAs (miRNAs) based Biomarkers for Acute Myocardial Infarction (AMI) Diagnosis. J Clin Exp Cardiolog 3:e106.

43. Meder B, Keller A, Vogel B, Haas J, Sedaghat-Hamedani F, et al. (2011) MicroRNA signatures in total peripheral blood as novel biomarkers for acute myocardial infarction. Basic Res Cardiol 106: 13-23.

44. Tang Y, Poteh NA, Chiang A, Kim I (2013) MicroRNA Therapeutics for Cardiac Disease. Cardiol Pharmacol 2:e113.

45. Widera C, Gupta SK, Lorenzen JM, Bang C, Bauersachs J, et al. (2011) Diagnostic and prognostic impact of six circulating microRNAs in acute coronary syndrome. J Mol Cell Cardiol 51: 872-875.

46. Thum T, Gross C, Fiedler J, Fischer T, Kissler S, et al. (2008) MicroRNA-21 contributes to myocardial disease by stimulating MAP kinase signalling in fibroblasts. Nature 456: 980-984.

47. Diehl P, Fricke A, Sander L, Stamm J, Bassler N, et al. (2012) Microparticles: major transport vehicles for distinct microRNAs in circulation. Cardiovasc Res 93: $633-644$
48. Ward JA, Esa N2, Pidikiti R2, Freedman JE2, Keaney JF2, et al. (2013) Circulating Cell and Plasma microRNA Profiles Differ between Non-STSegment and ST-Segment-Elevation Myocardial Infarction. Fam Med Med Sci Res 2: 108.

49. Rameshwar P (2013) Mesenchymal Stem Cells can be a Conduit for the Delivery of Therapeutic MicroRNA. J Stem Cell Res Ther 3:e112

50. Yang X1, Hou J, Han Z, Wang Y, Hao C, et al. (2013) One cell, multiple roles: contribution of mesenchymal stem cells to tumor development in tumor microenvironment. Cell Biosci 3: 5.

51. Zhou Q, Eades G1 (2012) MicroRNA Regulatory Networks Provide Feedback Mechanisms for Steroid Receptor Signaling. J Steroids Horm Sci 3.

52. Yang X, Li Z, Su Z, Davis K, Chen T, et al. (2011) Urinary Micrornas as Noninvasive Biomarkers for Acetaminophen-Induced Liver Injury. J Postgenom Drug Biomark Develop 1:101.

53. Yang $X$ (2012) Use Circulating microRNAs as Biomarkers of Drug- Induced Liver Injury. J Vaccines Vaccin 3:e105.

54. Lee CH, Chae JI, Ko HY, Kim S (2013) Molecular Beacon-Based Microrna Biosensor for Imaging EPC-Treated Cellular Therapy of Ischemia. J Mo Imaging Dynam 2:113.

55. Al-Shorafa H, Sharif FA (2012) MicroRNA in a Case of Unexplained Recurrent Pregnancy Loss. J Clin Case Rep 2:238.

56. Shahzad A, Williams LM, Lass-Flörl C, Shen A, Sami M (2012) MicroRNAs (miRNAs) In Virology: A Promising Transnational Research Approach. Trans Med 2:e110.

57. Shi G, Cui Q, Zhang Y (2009) MicroRNA Set : A Novel Way to Uncover the Potential Black Box of Chronic Heart Failure in MicroRNA Microarray Anal. 